\title{
LETTERS \\ Moral Distress Amongst Physician Trainees Regarding Futile Treatments
}

\author{
Elizabeth Dzeng, $M D, P h D, M P H^{1,2,3}$ \\ 'Division of Hospital Medicine, University of California, San Francisco, San Francisco, CA, USA; ${ }^{2}$ Department of Social and Behavioral Science, \\ Sociology Program, University of California, San Francisco, CA, USA; ${ }^{3}$ Primary Care Unit, Department of Public Health and Primary Care, University of \\ Cambridge, Cambridge, UK.
}

J Gen Intern Med 31(8):830

DOI: $10.1007 / \mathrm{s} 11606-016-3648-8$

(c) Society of General Internal Medicine 2016

I thank Dr. Adams for her interest and engagement with our article on physician trainee moral distress regarding potentially futile treatments at the end of life ${ }^{1}$. The causes of moral distress in the resident experience are multifaceted and complex. Contributing factors include systems-based elements, individual factors, institutional culture, and the learners' medical knowledge base, among others.

Dr. Adams hones in on one of these factors - that of prognostication, which is itself rife with uncertainty, even in the face of evidence-based medicine and experience ${ }^{2}$. I agree that the inpatient-centric nature of residency training and the fragmentation of longitudinal follow-up pose challenges to understanding the bigger picture.

I recall considerable reflection around my own understanding of potentially futile care after seeing a patient walk into clinic following a prolonged ICU course that all her doctors had believed she would not survive. These success stories are what make physicians' careers worthwhile. I believe that integrating opportunities to bear witness to their patients' post-ICU experience would be a beneficial addition to the residency experience and would provide residents with a necessary broader perspective.

However, to offer a balanced picture, residents would also need to be exposed to patients who had poor outcomes. A visit to a long-term acute care facility or nursing facility, for example, might reveal permanently bed-bound patients who are unable to be weaned from a ventilator or those who have suffered catastrophic brain injuries, subjecting them to what some describe, "a fate worse than death $^{3}$." This longitudinal awareness might result in more moral distress rather than less.
Focusing on residents' knowledge base, however, delegitimizes the residents' individual perception and beliefs, and detracts attention from the reality that moral distress can be a significant part of the residents' experience. Interventions could focus on creating a culture of openness towards ethical and emotional debriefing following difficult cases. Prospective preparation throughout medical training would help equip trainees with the ethical vocabulary necessary to reflect upon serious moral issues surrounding death and dying. I believe we owe it to the young physicians we train to help them become not only clinically competent physicians, but also ethical and compassionate carers.

Corresponding Author: Elizabeth Dzeng, MD, PhD, MPH; Division of Hospital Medicine, University of California, San Francisco, 533 Parnassus Avenue, Box 0131, San Francisco, CA, 94143-0131, USA (e-mail: dzeng@stanfordalumni.org).

\section{Compliance with Ethical Standards:}

Contributors: The author would like to thank David Levine, MD, MHS, $\mathrm{ScD}$, for a helpful discussions on this topic.

Funders: No funding was used for this manuscript.

Prior presentations: This paper has not been presented previously.

Conflict of Interest: There are no disclosures or conflicts of interest to report.

\section{REFERENCES}

1. Dzeng E, Colaianni A, Roland M, et al. Moral Distress Amongst American Physician Trainees Regarding Futile Treatments at the End of Life: A Qualitative Study. J Gen Intern Med. 2016;31(1):93-99. doi:10.1007/ s11606-015-3505-1.

2. Christakis N. Death Foretold: Prophecy and Prognosis in Medical Care. Chicago: University of Chicago Press; 2001.

3. Kitzinger J, Kitzinger C. The "window of opportunity" for death after severe brain injury : family experiences. Sociol Health Illn. 2013;35(7):10951112. doi:10.1111/1467-9566.12020. 